\title{
ON THE MAPPING CLASS GROUP OF A HEEGAARD SPLITTING
}

\author{
CHARALAMPOS CHARITOS and IOANNIS PAPADOPERAKIS \\ Agricultural University of Athens, Iera Odos 75, Athens 118 55, Greece \\ e-mails:bakis@aua.gr,papadoperakis@aua.gr
}

\section{GEORGIOS TSAPOGAS}

University of the Aegean, Samos 832 00, Greece

e-mail: get@aegean.gr

(Received 5 June 2012; revised 10 February 2013; accepted 10 May 2013; first published online 13 August 2013)

\begin{abstract}
For the mapping class group of 3-manifold with respect to a Heegaard splitting, a simplicial complex is constructed such that its group of automorphisms is identified with the mapping class group.
\end{abstract}

2010 Mathematics Subject Classification. 57N10, 57N35.

1. Introduction. For a closed 3-manifold $M$ with a fixed Heegaard splitting of genus $g$, notation $M^{3}=H_{g} \cup_{\Sigma_{g}} H_{g}^{\prime}$ with $\Sigma_{g}=\partial H_{g}=\partial H_{g}^{\prime}$, consider the group of homeomorphisms of $M$ which preserve the Heegaard splitting. By regarding, as usual, two such homeomorphisms as equivalent if there is an isotopy from one to the other via isotopies that preserve $H_{g}$ (and thus, $H_{g}^{\prime}$ ), we obtain a group which is naturally called the mapping class group of the Heegaard splitting of $M^{3}$, notation $\mathcal{M C G}\left(M^{3}, H_{g}\right)$.

In 1933, Goeritz [5] showed that the mapping class group $\mathcal{M C G}\left(\mathbb{S}^{3}, H_{2}\right)$ of the standard genus 2 Heegaard splitting of the 3-sphere is finitely generated. Scharlemann in [12] gave a modern proof of Georitz's result, and Akbas in [1] refined his argument to obtain a finite presentation of the mapping class group $\mathcal{M C G}\left(\mathbb{S}^{3}, H_{2}\right)$. Also, Cho in [3] recovered Akbas's result using a subcomplex of the disk complex of the handlebody of the splitting.

For genus $g \geq 3$ the question of finite generation of the mapping class group $\mathcal{M C G}\left(M^{3}, H_{g}\right)$ is open even in the case $M=\mathbb{S}^{3}$ (Scharlemann found serious gaps in the proofs of the above statement presented several years ago).

In this work we define a simplicial complex analogous to the curve complex for surfaces and show that the group of automorphisms of this complex is isomorphic to the mapping class group $\mathcal{M C G}\left(M^{3}, H_{g}\right)$, provided that $g \geq 3$. The construction of this complex builds on earlier work on the complex of incompressible surfaces for handlebodies defined in [2]. For the case $g=2$, we provide simple examples of automorphisms which are not geometric.

2. Definitions and statements of results. For a compact surface $S$, the complex of curves $\mathcal{C}(S)$, introduced by Harvey in [6], has vertices of isotopy classes of essential, non-boundary-parallel simple closed curves in $S$. A collection of vertices spans a simplex exactly when any two of them may be represented by disjoint curves, or 
equivalently when there is a collection of representatives for all of them, any two of which are disjoint. Analogously, for a 3-manifold $M$, the disk complex $\mathcal{D}(M)$ is defined by using the proper isotopy classes of compressing disks for $M$ as vertices. It was introduced in [11], where it was used in the study of mapping class groups of 3-manifolds. In [10], it was shown to be a quasi-convex subset of $\mathcal{C}(\partial M)$.

By $H_{g}$ we denote a 3-dimensional handlebody of genus $g \geq 2$. Recall that a compact connected surface $S \subset H_{g}$ with boundary is properly embedded if $S \cap \partial H_{g}=\partial S$ and $S$ is transversed to $\partial H_{g}$. A compressing disk for $S$ is an embedded disk $D$ such that $\partial D \subset S$ and $\partial D$ is essential in $S$. A properly embedded surface $S \subset H_{g}$ is incompressible if there are no compressing disks for $S$. Also recall that a map $F: S \times[0,1] \rightarrow H_{g}$ is a proper isotopy if for all $t \in[0,1],\left.F\right|_{S \times\{t\}}$ is a proper embedding. In this case we will say that $F(S \times\{0\})$ and $F(S \times\{1\})$ are properly isotopic in $H_{g}$, and we will use the symbol $\simeq$ to indicate isotopy in all cases (curves, surfaces etc) and the symbol $[S]$ to denote the isotopy class of $S$. We recall the following definition from [2].

DEFINITION. Let $\mathcal{I}\left(H_{g}\right)$ be a simplicial complex whose vertices are the proper isotopy classes of compressing disks for $\partial H_{g}$ and properly embedded boundary-parallel incompressible annuli and pairs of pants in $H_{g}$. For a vertex $[S]$, which is not a class of compressing disks, it is also required that $S$ is isotopic to a surface $\bar{S}$ embedded in $\partial H_{g}$ via an isotopy

$$
F: S \times[0,1] \rightarrow H_{g}
$$

with $F(S \times\{0\})=S, F(S \times\{1\})=\bar{S}$ and $F$ being proper when restricted to $[0,1)$. A collection of vertices spans a simplex in $\mathcal{I}\left(H_{g}\right)$ when any two of them may be represented by disjoint surfaces in $H_{g}$.

Observe that there do exist properly embedded pairs of pants that are not isotopic to a surface entirely contained in $\partial H_{g}$. We may regard $\mathcal{D}\left(H_{g}\right)$ as a subcomplex of $\mathcal{I}\left(H_{g}\right)$ or, by taking boundaries of the representative disks, $\mathcal{C}\left(\partial H_{g}\right)$. Also note that the vertices of $\mathcal{I}\left(H_{g}\right)$ represented by annuli exactly correspond to the vertices of $\mathcal{C}\left(\partial H_{g}\right)$ represented by curves that are essential in $\partial H_{g}$ but are not meridian boundaries. We define the complex of annuli $\mathcal{A}\left(H_{g}\right)$ to be the subcomplex of $\mathcal{I}\left(H_{g}\right)$ spanned by these vertices. Together, the vertices of $\mathcal{D}\left(H_{g}\right) \cup \mathcal{A}\left(H_{g}\right)$ span a copy of $\mathcal{C}\left(\partial H_{g}\right)$ in $\mathcal{I}\left(H_{g}\right)$, and we regard $\mathcal{C}\left(\partial H_{g}\right)$ as a subcomplex of $\mathcal{I}\left(H_{g}\right)$. We will denote by $\mathcal{D}($ resp. $\mathcal{A})$ the vertex set of $\mathcal{D}\left(H_{g}\right)$ (resp. $\mathcal{A}\left(H_{g}\right)$ ). A vertex in $\mathcal{D}($ resp. $\mathcal{A})$ will be called a meridian (resp. annular) vertex. The vertex set of $\mathcal{I}\left(H_{g}\right) \backslash\left(\mathcal{D}\left(H_{g}\right) \cup \mathcal{A}\left(H_{g}\right)\right)$ will be denoted by $\mathcal{P}$ and a vertex in $\mathcal{P}$ will be called a pants vertex. Observe that a vertex $v$ in either $\mathcal{D}$ or $\mathcal{A}$ determines a unique, up to isotopy, simple closed curve in $\partial H_{g}$, which will be called the boundary curve of $v$, denoted by $\partial v$. Similarly, a vertex in $\mathcal{P}$ determines uniquely, up to isotopy, a pair or a triple of mutually disjoint simple closed curves in $\partial H_{g}$.

REMARK 1. The complex $\mathcal{I}\left(H_{g}\right)$ can be thought of in the following way: Take the curve complex $\mathcal{C}\left(\partial H_{g}\right)$ and add a vertex for every pair $\left(\alpha_{1}, \alpha_{2}\right)$ or triple $\left(\alpha_{1}, \alpha_{2}, \alpha_{3}\right)$ of non-meridian simple closed curves which bound a pair of pants in $\partial H_{g}$. Then add an edge from the new vertex to the vertices $\alpha_{i}$ as well as to any other vertex in $\mathcal{C}\left(\partial H_{g}\right)$ disjoint from $\alpha_{i}$ 's. In particular, the new vertices are connected to (some) meridian vertices. By construction, such a complex cannot be isomorphic to any kind of subdivision of $\mathcal{C}\left(\partial H_{g}\right)$. For example, subdivisions do not alter dimension, whereas 


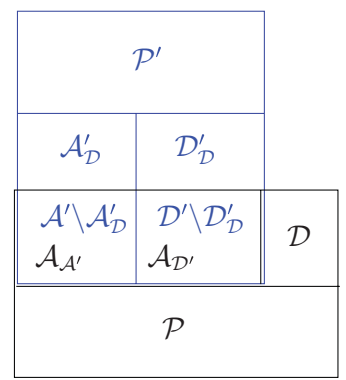

Figure 1. (Colour online) The vertex sets in $\mathcal{I}\left(M, H_{g}\right)$.

$\mathcal{I}\left(H_{g}\right)$ is not homogeneous with respect to dimension (see properties preceding Lemma 5).

In an identical way the complex $\mathcal{I}\left(H_{g}^{\prime}\right)$ is defined and we use the notation $\mathcal{P}^{\prime}$ (resp. $\left.\mathcal{A}^{\prime}, \mathcal{D}^{\prime}\right)$ for the vertex set of $\mathcal{I}\left(H_{g}^{\prime}\right) \backslash\left(\mathcal{D}\left(H_{g}^{\prime}\right) \cup \mathcal{A}\left(H_{g}^{\prime}\right)\right)\left(\operatorname{resp} . \mathcal{A}\left(H_{g}^{\prime}\right), \mathcal{D}\left(H_{g}^{\prime}\right)\right)$.

Observe that an essential simple closed curve in $\Sigma_{g}=\partial H_{g}=\partial H_{g}^{\prime}$ determines a unique vertex in $\mathcal{I}\left(H_{g}\right)$ (annular or meridian) and a unique vertex in $\mathcal{I}\left(H_{g}^{\prime}\right)$ (possibly of different type). We will also use the following notation:

$\mathcal{D}_{\mathcal{D}}^{\prime}:=\left\{v \in \mathcal{D}^{\prime} \mid\right.$ the boundary curve of $v$ is a meridian in $\left.H_{g}\right\}$,

$\mathcal{A}_{\mathcal{D}}^{\prime}:=\left\{v \in \mathcal{A}^{\prime} \mid\right.$ the boundary curve of $v$ is a meridian in $\left.H_{g}\right\}$,

$\mathcal{A}_{\mathcal{A}^{\prime}}:=\left\{v \in \mathcal{A} \mid\right.$ the boundary curve of $v$ is nonmeridian in $\left.H_{g}^{\prime}\right\}$,

$\mathcal{A}_{\mathcal{D}^{\prime}}:=\left\{v \in \mathcal{A} \mid\right.$ the boundary curve of $v$ is a meridian in $\left.H_{g}^{\prime}\right\}$.

We define a simplicial complex $\mathcal{I}\left(M, H_{g}\right)$ for the manifold $M$ with respect to the Heegaard splitting $M^{3}=H_{g} \cup_{\Sigma_{g}} H_{g}^{\prime}$ by identifying $\mathcal{I}\left(H_{g}\right)$ with $\mathcal{I}\left(H_{g}^{\prime}\right)$ along the vertex set $\mathcal{A}$ of $\mathcal{I}\left(H_{g}\right)$ as follows.

DEFINITION 2. Let $\mathcal{I}\left(M, H_{g}\right)$ be the simplicial complex whose

- vertices are all vertices in $\mathcal{I}\left(H_{g}\right) \cup \mathcal{I}\left(H_{g}^{\prime}\right)$ with the exception that a vertex $u$ in $\mathcal{D}^{\prime} \backslash \mathcal{D}_{\mathcal{D}}^{\prime}$ (resp. $\mathcal{A}^{\prime} \backslash \mathcal{A}_{\mathcal{D}}^{\prime}$ ) is identified with the corresponding vertex $u^{\prime}$ in $\mathcal{A}_{\mathcal{D}^{\prime}}$ (resp. $\mathcal{A}_{\mathcal{A}^{\prime}}$ ), that is, with the unique vertex $u^{\prime}$ in $\mathcal{A}_{\mathcal{D}^{\prime}}\left(\right.$ resp. $\mathcal{A}_{\mathcal{A}^{\prime}}$ ) for which $\partial u^{\prime}$ is isotopic to $\partial u$ in $\Sigma_{g}$

- edges are all edges in $\mathcal{I}\left(H_{g}\right) \cup \mathcal{I}\left(H_{g}^{\prime}\right)$ with the exception that each edge $(u, v)$ in $\mathcal{I}\left(H_{g}\right)$ with endpoints $u, v \in \mathcal{A}$ is identified with the (corresponding) edge in $\mathcal{I}\left(H_{g}^{\prime}\right)$ with endpoints $u^{\prime} \equiv u, v^{\prime} \equiv v \in\left(\mathcal{D}^{\prime} \backslash \mathcal{D}_{\mathcal{D}}^{\prime}\right) \cup\left(\mathcal{A}^{\prime} \backslash \mathcal{A}_{\mathcal{D}}^{\prime}\right)$.

Then $\mathcal{I}\left(M, H_{g}\right)$ is the flag complex with the above vertices and edges, that is, if all the edges of a potential face belong to the complex, then that face is required to belong to the complex.

We will be viewing both $\mathcal{I}\left(H_{g}\right)$ and $\mathcal{I}\left(H_{g}^{\prime}\right)$ as subcomplexes of $\mathcal{I}\left(M, H_{g}\right)$. In the vertex set of $\mathcal{I}\left(M, H_{g}\right)$ we clearly have

$$
\mathcal{A}_{\mathcal{A}^{\prime}} \cup \mathcal{A}_{\mathcal{D}^{\prime}}=\mathcal{A}, \mathcal{D}_{\mathcal{D}}^{\prime} \cup \mathcal{A}_{\mathcal{D}^{\prime}}=\mathcal{D}^{\prime} \text { and } \mathcal{A}_{\mathcal{A}^{\prime}} \cup \mathcal{A}_{\mathcal{D}}^{\prime}=\mathcal{A}^{\prime}
$$

The above notation is summarized in Figure 1. 
REMARK 3. It would be plausible to define $\mathcal{I}\left(M, H_{g}\right)$ by identifying the copies of $\mathcal{C}\left(\partial H_{g}\right)$ found inside $\mathcal{I}\left(H_{g}\right)$ and $\mathcal{I}\left(H_{g}^{\prime}\right)$. However, such a complex does not serve our purposes because the pant subcomplexes $\mathcal{P}, \mathcal{P}^{\prime}$ are not connected and, thus, an automorphism of $\mathcal{I}\left(M, H_{g}\right)$ may not preserve them in the sense exhibited in Example 4.

Our goal is to show that for any closed 3-manifold $M$ with a fixed Heegaard splitting of genus $g \geq 3$, the automorphisms of the complex $\mathcal{I}\left(M, H_{g}\right)$ are all geometric, that is, they are induced by homeomorphisms of $M$ that preserve the Heegaard splitting. This can be rephrased by saying that the map

$$
A: \operatorname{MCG}\left(M, H_{g}\right) \rightarrow \operatorname{Aut}\left(\mathcal{I}\left(M, H_{g}\right)\right)
$$

is an onto map where $\operatorname{Aut}\left(\mathcal{I}\left(M, H_{g}\right)\right)$ is a group of automorphisms of the complex $\mathcal{I}\left(M, H_{g}\right)$. Moreover, we will show (see Theorem 10) that the map $A$ is $1-1$.

For the proof of this result we first show that the dimension of the link of a vertex of $\mathcal{I}\left(M, H_{g}\right)$ lying in $\mathcal{A}$ is distinct (in fact, bigger) than the dimension of the link of any other vertex of $\mathcal{I}\left(M, H_{g}\right)$ not contained in $\mathcal{A}$. An important step is to establish that an automorphism $\phi$ of $\mathcal{I}\left(M, H_{g}\right)$ must map each vertex $v$ in $\mathcal{P}$ to a vertex $f(v)$ which also belongs to $\mathcal{P}$ (provided that $M$ is not homeomorphic to the connected sum of copies of $\left.\mathbb{S}^{2} \times \mathbb{S}^{1}\right)$ and similarly for $\mathcal{D}$. In showing this, we use the notion of the pants complex, introduced by Hatcher and Thurston in [8] and its connectivity properties (see [7]). Finally, we use the corresponding result for handlebodies shown in [2], namely, that $\mathcal{M C G}\left(H_{g}\right)$ is isomorphic to $\operatorname{Aut}\left(\mathcal{I}\left(H_{g}\right)\right)$.

If $v$ is a vertex in $\mathcal{I}\left(M, H_{g}\right)$, we will denote by $L k(v)$ the link of the vertex $v$ in $\mathcal{I}\left(M, H_{g}\right)$, namely, for each simplex $\sigma$ containing $v$ consider the faces of $\sigma$ not containing $v$ and take the union over all such $\sigma$. We will use the notation $¥$ to declare that two links are not isomorphic as complexes.

We will also use the classical notation $\Sigma_{n, b}$ to denote the surface of genus $n$ with $b$ boundary components.

We conclude this section by demonstrating an example which shows that in the case $g=2$, non-geometric automorphisms of $\mathcal{I}\left(M, H_{g}\right)$ may exist.

EXAMPLE 4. Let $M=H_{2} \cup_{\Sigma} H_{2}^{\prime}$, where $\Sigma=\partial H_{g}=\partial H_{g}^{\prime}$ is the genus 2 closed surface. One may think of $M$ as the 3-sphere with the standard Heegaard splitting. Choose a non-separating essential simple closed curve $\alpha$ in $\Sigma$ which is not a generator for $\pi_{1}\left(H_{2}\right)$ (for example, choose $\alpha$ to represent the second power of a generator of $\left.\pi_{1}\left(H_{2}\right)\right)$. Similarly, choose $\beta$ in $\Sigma$ which is not a generator for $\pi_{1}\left(H_{2}^{\prime}\right)$ and, in addition, $\alpha \cap \beta=\varnothing$. Then choose a non-separating essential simple closed curve $\gamma$ in $\Sigma$ such that

$$
\alpha \cap \gamma=\varnothing=\beta \cap \gamma
$$

Clearly, the curves $\alpha, \beta, \gamma$ decompose $\Sigma$ into two pairs of pants, denoted by $P_{1}, P_{2}$. Observe that $P_{1}, P_{2}$ are not isotopic in $H_{2}$. For, if $P_{1}, P_{2}$ were isotopic in $H_{2}$, then $H_{2}$ would be homeomorphic to $P_{1} \times[0,1]$ making $\alpha$ a generator for $\pi_{1}\left(H_{2}\right)$, a contradiction by choice. Similarly, $P_{1}, P_{2}$ are not isotopic in $H_{2}^{\prime}$. Thus, the complex $\mathcal{I}\left(M, H_{2}\right)$ contains distinct vertices $\left[P_{1}\right],\left[P_{2}\right] \in \mathcal{P}$ and $\left[P_{1}\right]^{\prime},\left[P_{2}\right]^{\prime} \in \mathcal{P}^{\prime}$. Observe that $\left[P_{1}\right]$ is connected by an edge only with the vertices $[\alpha],[\beta],[\gamma],\left[P_{2}\right]$ and similarly for 
$\left[P_{1}\right]^{\prime}$. Let $\phi$ be the automorphism of $\mathcal{I}\left(M, H_{2}\right)$ defined by

$$
\phi\left(\left[P_{i}\right]\right)=\left[P_{i}\right]^{\prime} \text { and } \phi\left(\left[P_{i}\right]^{\prime}\right)=\left[P_{i}\right]
$$

and $\phi(v)=v$ for all $v \neq\left[P_{i}\right],\left[P_{i}\right]^{\prime}, i=1,2$.

If $\phi$ were geometric, then, since $\phi$ is the identity on $\mathcal{C}(\Sigma), \phi$ would have to be induced by a homeomorphism $F: M \rightarrow M$ with $\left.F\right|_{\Sigma}$ being the identity. As any homeomorphism $\Sigma \rightarrow \Sigma$ extends uniquely to the handlebody it bounds, $F$ would have to be the identity on $M$.

3. Properties of the complex $\mathcal{I}\left(M, H_{g}\right)$. In this section we will calculate the dimension of the link of all types of vetrices in $\mathcal{I}\left(M, H_{g}\right)$. Although most properties hold for $g=2$, we will assume throughout this section that $g \geq 3$. We recall certain properties from [2]:

(DM) If $v$ is a meridian vertex in $\mathcal{I}\left(H_{g}\right)$ then its link in $\mathcal{I}\left(H_{g}\right)$ has dimension $5 g-9$ (Lemma 4).

(DP) If $v$ is a pants vertex in $\mathcal{I}\left(H_{g}\right)$ then its link in $\mathcal{I}\left(H_{g}\right)$ has dimension $5 g-7$ (Proposition 2).

(DA) If $v$ is an annular vertex in $\mathcal{I}\left(H_{g}\right)$ then its link in $\mathcal{I}\left(H_{g}\right)$ has dimension $5 g-7$ (Lemma 3).

Identical properties hold for the vertices in $\mathcal{I}\left(H_{g}^{\prime}\right)$. Analogous properties hold in the complex $\mathcal{I}\left(M, H_{g}\right)$.

LEMMA 5. If $v \in \mathcal{D} \cup \mathcal{D}_{\mathcal{D}}^{\prime}$ then its link in $\mathcal{I}\left(M, H_{g}\right)$ has dimension $5 g-9$. If $v \in \mathcal{A}_{\mathcal{D}}^{\prime} \cup \mathcal{P} \cup \mathcal{P}^{\prime}$ then its link in $\mathcal{I}\left(M, H_{g}\right)$ has dimension $5 g-7$.

Proof. It is straightforward since, by the definition of $\mathcal{I}\left(M, H_{g}\right)$, the link of a vertex $v \in \mathcal{D} \cup \mathcal{P}$ in $\mathcal{I}\left(M, H_{g}\right)$ is identical with the link of $v$ in $\mathcal{I}\left(H_{g}\right)$. Similarly, the link of a vertex $v \in \mathcal{D}_{\mathcal{D}}^{\prime} \cup \mathcal{A}_{\mathcal{D}}^{\prime} \cup \mathcal{P}^{\prime}$ in $\mathcal{I}\left(M, H_{g}^{\prime}\right)$ is identical with the link of $v$ in $\mathcal{I}\left(H_{g}^{\prime}\right)$.

We next examine the dimension of the link of the vertices in $\mathcal{A}=\mathcal{A}_{\mathcal{A}^{\prime}} \cup \mathcal{A}_{\mathcal{D}^{\prime}}$.

Lemma 6. If $v \in \mathcal{A}_{\mathcal{A}^{\prime}}$, then the dimension of $\operatorname{Lk}(v)$ in $\mathcal{I}\left(M, H_{g}\right)$ is $\geq 7 g-9$. If $v \in \mathcal{A}_{\mathcal{D}^{\prime}}$, then the dimension of $\operatorname{Lk}(v)$ in $\mathcal{I}\left(M, H_{g}\right)$ is $\geq 5 g-6$.

Proof. By property (DA) we have that $v \in \mathcal{A}$ is contained in a simplex of dimension $5 g-6$ lying entirely in $\mathcal{I}\left(H_{g}\right) \subset \mathcal{I}\left(M, H_{g}\right)$.

Let $v \in \mathcal{A}_{\mathcal{A}^{\prime}}$. There exist $3 g-2$ simple closed curves $\beta_{1}, \ldots \beta_{3 g-2}$ in $\Sigma_{g}=\partial H_{g}^{\prime}$ such that $\left\{\partial v, \beta_{1}, \ldots \beta_{3 g-2}\right\}$ is a pants decomposition for $\Sigma_{g}$ and each $\beta_{i}$ is non-meridian in $H_{g}^{\prime}$. This implies that the pants decomposition $\left\{\partial v, \beta_{1}, \ldots \beta_{3 g-2}\right\}$ determines $2 g-2$ pairs of pants which are incompressible in $H_{g}^{\prime}$. Thus, there exist $2 g-2$ vertices in $\mathcal{P}^{\prime}$ which belong to $L k(v)$.

Let $v \in \mathcal{A}_{\mathcal{D}^{\prime}}$. As $g$ is assumed to be $\geq 3$, cutting $H_{g}^{\prime}$ along the meridian $v$ we always (i.e. $v$ separating or non-separating) obtain a handlebody of genus $\geq 2$ with one or two disks marked on its boundary (these being the disks bounded by copies of $\partial v$ ). On the boundary of this handlebody we may find non-meridian, simple, mutually disjoint curves $\gamma_{1}, \gamma_{2}, \gamma_{3}$ which form a pair of pants such that each $\gamma_{i}$ does not intersect with the marked boundary copies of $\partial v$. Figure 2. exhibits this in the case $g=3$ and $\partial v$ is non-separating. It follows that $\gamma_{1}, \gamma_{2}, \gamma_{3}$ determine a pants vertex $w^{\prime} \in \mathcal{P}^{\prime}$ which is connected by an edge with $v$ in $\mathcal{I}\left(H_{g}^{\prime}\right)$. This completes the proof of the Lemma. 


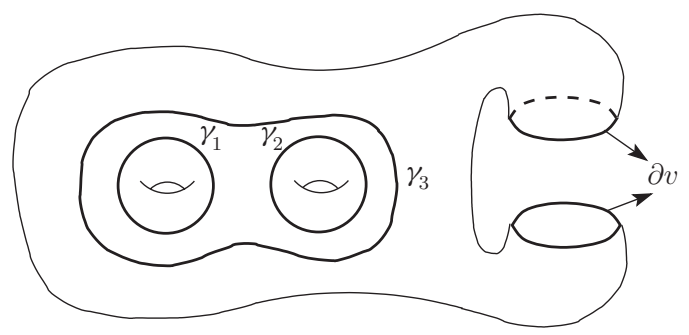

Figure 2.

We will need the following.

Lemma 7. If $\phi \in \operatorname{Aut}\left(\mathcal{I}\left(M, H_{g}\right)\right)$ and $v \in \mathcal{P}$, then $\phi(v) \notin \mathcal{A}_{\mathcal{D}}^{\prime}$.

Proof. Let $v \in \mathcal{P}$ and denote by $\beta$ one of the three boundary components of a pair of pants representing $v$. The 1 -skeleton of $L k(v)$ is a cone graph, that is, there exists a vertex which is connected by an edge with any other vertex in $L k(v)$ (the annular vertex $v_{\beta}$ with $\partial v_{\beta}=\beta$ is one such). We will reach a contradiction by showing that for any $u \in \mathcal{A}_{\mathcal{D}}^{\prime}$ the 1 -skeleton of $\operatorname{Lk}(u)$ is not a cone graph. For this it suffices to show that

$$
\forall w \in L k(u), \exists r \in L k(u): w, r \text { are not connected by an edge. }
$$

For, if $\beta_{w}$ is a boundary component of a surface representing $w \in L k(u)$, then there exists a curve $\gamma$ such that $\partial u \cap \gamma=\varnothing$ and $\gamma \cap \beta_{w} \neq \varnothing$. Let $r$ be the vertex in $\mathcal{D}^{\prime} \cup \mathcal{A}^{\prime}$ with $\partial r=\gamma$. Then $r \in \operatorname{Lk}(u)$ is the required vertex which is not connected by an edge with $w$.

PROPOSITION 8. If $\phi$ is an automorphism of $\mathcal{I}\left(M, H_{g}\right)$ then $v \in \mathcal{A}$ if and only if $\phi(v) \in \mathcal{A}$.

Proof. The conclusion is straightforward by dimension arguments based on Lemmas 5 and 6.

We conclude this section by showing the following property.

PROPOSITION 9. The subcomplex of $\mathcal{I}\left(M, H_{g}\right)$ spanned by the vertices $\mathcal{D} \cup \mathcal{P}$ is path-connected.

Proof. By the argument at the end of Lemma 6 , if $w \in \mathcal{D}$, there exists a pants vertex $u \in \mathcal{P}$ which is connected by an edge with $v$. Therefore, it suffices to consider two arbitrary vertices $u, v \in \mathcal{P}$ in order to exhibit path-connectedness of $\mathcal{D} \cup \mathcal{P}$.

We will use the notion of the pants complex for surfaces originally introduced by Hatcher and Thurston in [8]. We refer readers to [9, Section 2.2] for precise definition and properties. We briefly recall that the 1-skeleton of the pants complex of a (closed for us) surface $\Sigma_{g}$ (usually called the pants graph) has one vertex for each pants decomposition of $\Sigma_{g}$ (equivalently, for each maximal simplex 1 in $\mathcal{C}\left(\Sigma_{g}\right)$ ) and edges joining vertices whose associated pants decomposition differs by elementary moves. More precisely, two vertices $P=\left(\alpha_{1}, \ldots, \alpha_{3 g-3}\right)$ and $P^{\prime}$ span an edge if $P^{\prime}$ can be obtained from $P$ by replacing one curve in $P$, say $\alpha_{1}$, by another curve, say $\alpha_{1}^{\prime}$, such that the intersection number of $\alpha_{1}$ with $\alpha_{1}^{\prime}$ is 2 if they both belong to a subsurface of $\Sigma_{g}$ of type $\Sigma_{0,4}$ and the intersection number is 1 if they both belong to a subsurface of $\Sigma_{g}$ of type $\Sigma_{1,1}$. 
Apparently, for each pants vertex $v \in \mathcal{P}$ we may choose a pants decomposition $P_{v}$ such that the boundary curves of $v$ belong to $P_{v}$. It was shown in [7] that the pants complex is connected and simply connected. This means that for arbitrary vertices $u, v \in \mathcal{P}$ there exists pants decompositions $P_{0}=P_{u}, P_{1}, \ldots, P_{k-1}, P_{k}=P_{v}$ such that $P_{i}, P_{i+1}$ differ by an elementary move for $i=0, \ldots, k-1$. In particular, $P_{i}, P_{i+1}$ have $3 g-4$ curves in common. It is clear that for each $i=1, \ldots, k-2$ we may choose a pair of pants $p_{i}$ in $P_{i}$ such that $p_{i}, p_{i+1}$ have disjoint boundary components and similarly for $u, p_{1}$ and $p_{k-1}, v$. If all boundary components of all $p_{i}$ are non-meridians, the sequence $u, p_{1}, \ldots, p_{k-1}, v$ gives rise to path of vertices in $\mathcal{P}$ from $v$ to $u$ and we are done. If some $p_{i}$ is a compressible pair of pants in $H_{g}$, we may use a boundary curve of $p_{i}$ which is meridian.

\section{Proof of the main theorem.}

Let

$$
A: \operatorname{MCG}\left(M, H_{g}\right) \rightarrow \operatorname{Aut}\left(\mathcal{I}\left(M, H_{g}\right)\right)
$$

be the map sending a mapping class $F$ to the automorphism it induces on $\mathcal{I}\left(M, H_{g}\right)$, that is, $A(F)$ is given by

$$
A(F)[S]:=[F(S)],
$$

where $[S]$ denotes the isotopy class (vertex) determined by $S$.

THEOREM 10. Assume $M$ is not homeomorphic to the connected sum of copies of $\mathbb{S}^{2} \times \mathbb{S}^{1}$. Then the map $A: \mathcal{M C G}\left(M, H_{g}\right) \rightarrow \operatorname{Aut}\left(\mathcal{I}\left(M, H_{g}\right)\right)$ is an isomorphism for $g \geq 3$.

Proof. We will use the corresponding result, see [2, Theorem 7], applied to the handlebodies $H_{g}$ and $H_{g}^{\prime}$.

We first show that every $\phi \in \operatorname{Aut}\left(\mathcal{I}\left(M, H_{g}\right)\right)$ is geometric. We claim that either Case I: $\phi(\mathcal{D})=\mathcal{D}$ and $\phi(\mathcal{P})=\mathcal{P}$

or

Case II: $\phi(\mathcal{P} \cup \mathcal{D})=\mathcal{P}^{\prime} \cup \mathcal{D}_{\mathcal{D}}^{\prime}$, in which case $\mathcal{A}_{\mathcal{D}}^{\prime}=\varnothing$.

Let $v \in \mathcal{P}$. By dimension considerations (see Lemmas 5 and 6), we have $\phi(v) \in$ $\mathcal{P} \cup \mathcal{P}^{\prime} \cup \mathcal{A}_{\mathcal{D}}^{\prime}$, and by Lemma $7, \phi(v) \in \mathcal{P} \cup \mathcal{P}^{\prime}$.

Assume first that $\phi(v) \in \mathcal{P}$. By Proposition $9, \phi(w) \in \mathcal{P}$ for all $w \in \mathcal{P}$. To see the latter, assume that $\phi(w) \in \mathcal{P}^{\prime}$ for some $w \in \mathcal{P}$. Choose a path $\sigma$ from $v$ to $w$ whose vertices are in $\mathcal{P} \cup \mathcal{D}$. Then $\phi(\sigma)$ is a path from a vertex in $\mathcal{P}$ to a vertex in $\mathcal{P}^{\prime}$. It follows that some vertex of $\sigma$ is mapped to a vertex in $\mathcal{A}$, which is a contradiction by Proposition 8 . Thus, we have that if for an arbitrary $v \in \mathcal{P}, \phi(v) \in \mathcal{P}$ then $\phi(\mathcal{P})=\mathcal{P}$ and clearly $\phi(\mathcal{D})=\mathcal{D}$ as stated in Case I.

Now assume that $\phi(v) \in \mathcal{P}^{\prime}$. Using Proposition 9 in the same way as above, we have $\phi(\mathcal{P} \cup \mathcal{D}) \subseteq \mathcal{P}^{\prime} \cup \mathcal{D}_{\mathcal{D}}^{\prime} \cup \mathcal{A}_{\mathcal{D}}^{\prime}$. Then by dimension arguments (cf Lemma 5) we have $\phi(\mathcal{D})=\mathcal{D}_{\mathcal{D}}^{\prime}$ and $\phi(\mathcal{P})=\mathcal{P}^{\prime} \cup \mathcal{A}_{\mathcal{D}}^{\prime}$. By Lemma 7, we have $\phi(\mathcal{P})=\mathcal{P}^{\prime}$ and, again by dimension arguments, we have $\phi\left(\mathcal{A}_{\mathcal{D}}^{\prime}\right)=\mathcal{A}_{\mathcal{D}}^{\prime}$. The latter is impossible if $\mathcal{A}_{\mathcal{D}}^{\prime} \neq \varnothing$ : for, if $x \in \mathcal{A}_{\mathcal{D}}^{\prime}$ and $\phi(x) \in \mathcal{A}_{\mathcal{D}}^{\prime}$ we may choose a pair of pants $w \in \mathcal{P}^{\prime}$ in the $L k(\phi(x))$. Then 
$\phi^{-1}(w) \in L k(x)$ and $\phi^{-1}(w) \in \mathcal{P}$, a contradiction since $x \in \mathcal{A}_{\mathcal{D}}^{\prime}$ and no vertex in $\mathcal{A}_{\mathcal{D}}^{\prime}$ is connected by an edge with a vertex in $\mathcal{P}$. Thus, $\mathcal{A}_{\mathcal{D}}^{\prime}=\varnothing$ as stated in Case II.

We now proceed with the proof of the theorem in Case I. We have $\phi\left(\mathcal{P}^{\prime}\right)=\mathcal{P}^{\prime}$ and $\phi\left(\mathcal{D}^{\prime} \cup \mathcal{A}^{\prime}\right)=\mathcal{D}^{\prime} \cup \mathcal{A}^{\prime}$. Thus, $\phi$ induces an automorphism $\phi^{\prime}$ of $\mathcal{I}\left(H_{g}^{\prime}\right)$, and by [2, Theorem 7] $\phi^{\prime}$ is geometric, hence we obtain a homeomorphism $F^{\prime}: H_{g}^{\prime} \rightarrow H_{g}^{\prime}$ realizing $\phi^{\prime}$. Such a homeomorphism $F^{\prime}$ is unique. Since $\phi^{\prime}\left(\mathcal{D}_{\mathcal{D}}^{\prime} \cup \mathcal{A}_{\mathcal{D}}^{\prime}\right)=\mathcal{D}_{\mathcal{D}}^{\prime} \cup \mathcal{A}_{\mathcal{D}}^{\prime}$, it follows that $F^{\prime}$ maps each simple closed curve in $\Sigma=\partial H_{g}^{\prime}=\partial H_{g}$ which bounds a meridian in $H_{g}$ to another such meridian. Therefore, $F^{\prime}$ extends to a homeomorphism of $H_{g}$. This extension is unique (see, for example, [4, Theorem 3.7 p. 94]). In other words, $F^{\prime}$ defines a homeomorphism

$$
F_{M}: H_{g} \cup_{\Sigma_{g}} H_{g}^{\prime} \rightarrow H_{g} \cup_{\Sigma_{g}} H_{g}^{\prime} .
$$

Clearly, the composition $A\left(F_{M}^{-1}\right) \circ \phi$ is an automorphism of $\mathcal{I}\left(M, H_{g}\right)$, which is the identity on $\mathcal{I}\left(H_{g}^{\prime}\right)$. Thus, we may assume that the automorphism $\phi \in$ $\operatorname{Aut}\left(\mathcal{I}\left(M, H_{g}\right)\right)$ is the identity on $\mathcal{I}\left(H_{g}^{\prime}\right)$ and we want to show that it is the identity on the whole complex $\mathcal{I}\left(M, H_{g}\right)$.

We first show that $\phi$ is the identity on $\mathcal{D}$. Let $w \in \mathcal{D}$, and let $D$ be a meridian in $H_{g}$ representing $w$. If $\phi(w) \neq w$, that is, $\phi(w)$ is represented by a meridian $D^{\prime}$ non-isotopic to $D$, then we may find a simple, essential curve $\alpha$ in $\partial H_{g}$ which does not bound a meridian in $H_{g}$ such that $\partial D \cap \alpha \neq \varnothing$ and $\partial D^{\prime} \cap \alpha=\varnothing$. Since $\phi$ fixes the vertex represented by $\alpha$, we have a contradiction. Thus, $\phi$ fixes every vertex $w \in \mathcal{D}$.

It follows that $\phi$ induces an automorphism $\left.\phi\right|_{\mathcal{I}\left(H_{g}\right)}$ of $\mathcal{I}\left(H_{g}\right)$ which fixes $\mathcal{A} \cup \mathcal{D}$. This automorphism is geometric (see [2, Theorem 7]), that is, there exists a homeomorphism $G: H_{g} \rightarrow H_{g}$ realizing $\left.\phi\right|_{\mathcal{I}\left(H_{g}\right)}$. As $\left.\phi\right|_{\mathcal{I}\left(H_{g}\right)}$ fixes every vertex in $\mathcal{A} \cup \mathcal{D}, G$ is is the identity on $\Sigma=\partial H_{g}$. As every homeomorphism of $\partial H_{g}$ which extends to a homeomorphism of $H_{g}$ it does so uniquely, it follows that $G$ is the identity. Therefore, $\left.\phi\right|_{\mathcal{I}\left(H_{g}\right)}$ is the identity on $\mathcal{I}\left(H_{g}\right)$ and, thus, is the identity on the whole complex $\mathcal{I}\left(M, H_{g}\right)$ as required. This completes the proof in Case I.

We proceed with Case II. As $\mathcal{A}_{\mathcal{D}}^{\prime}=\varnothing$, we have

Case IIa: $\mathcal{D}^{\prime} \backslash \mathcal{D}_{\mathcal{D}}^{\prime} \neq \varnothing$, and

Case IIb: $\mathcal{D}^{\prime} \backslash \mathcal{D}_{\mathcal{D}}^{\prime}=\varnothing$, that is, $\mathcal{D}_{\mathcal{D}}^{\prime} \cap \mathcal{A}=\varnothing$.

We will show that Case IIa does not occur, and in Case IIb $M$ is homeomorphic to the connected sum of copies of $\mathbb{S}^{2} \times \mathbb{S}^{1}$. Let $w \in \mathcal{A}^{\prime}=\mathcal{A}_{\mathcal{A}^{\prime}}$. Then $\operatorname{Lk}(w)$ contains $2 g-2$ pant vertices in $\mathcal{P}$, which form a simplex, and similarly $2 g-2$ pant vertices in $\mathcal{P}^{\prime}$. This implies that $\phi(w) \notin \mathcal{D}^{\prime} \backslash \mathcal{D}_{\mathcal{D}}^{\prime}$ because a meridian vertex in $\mathcal{I}\left(H_{g}^{\prime}\right)$ cannot have $2 g-2$ pant vertices from $\mathcal{P}^{\prime}$ in its link. It follows that $\phi\left(\mathcal{A}_{\mathcal{A}^{\prime}}\right)=\mathcal{A}_{\mathcal{A}^{\prime}}$ and $\phi\left(\mathcal{D}^{\prime} \backslash \mathcal{D}_{\mathcal{D}}^{\prime}\right)=$ $\mathcal{D}^{\prime} \backslash \mathcal{D}_{\mathcal{D}}^{\prime}$. Let now $v \in \mathcal{D}^{\prime} \backslash \mathcal{D}_{\mathcal{D}}^{\prime}$ and denote by $p_{1}, \ldots, p_{2 g-2}$ a maximal set of pant vertices from $\mathcal{P}$ contained in $\operatorname{Lk}(v)$. As $\phi\left(p_{i}\right)=p_{i}^{\prime}$ with $p_{i}^{\prime} \in \mathcal{P}^{\prime}$, we have a contradiction because $\phi(v) \in \mathcal{D}^{\prime} \backslash \mathcal{D}_{\mathcal{D}}^{\prime}$ and $\partial \phi(v)$ bounds a meridian in $H_{g}^{\prime}$ (thus, $\phi(v)$ cannot have $2 g-2$ pant vertices from $\mathcal{P}^{\prime}$ in its link). This shows that Case IIa cannot occur.

We conclude the proof of the theorem by observing that in Case IIb the manifold $M$ is homeomorphic to the connected sum of copies of $\mathbb{S}^{2} \times \mathbb{S}^{1}$. If $H_{2}=\mathbb{D}^{2} \times \mathbb{S}^{1}$ is glued with $H_{2}^{\prime}=\mathbb{D}^{2} \times \mathbb{S}^{1}$ along $\mathbb{S}^{1} \times \mathbb{S}^{1}$ so that every curve which is a meridian boundary in $H_{2}$ is identified with a meridian boundary in $H_{2}^{\prime}$ then $M$ is homeomorphic to $\mathbb{S}^{2} \times \mathbb{S}^{1}$. Inductively, if $a$ is a separating curve in $\partial H_{g}=\partial H_{g}^{\prime}$ which bounds a meridian $D_{\alpha}$ in $H_{g}$ and a meridian $D_{\alpha}^{\prime}$ in $H_{g}^{\prime}$, then cutting along the 2-sphere $D_{a} \cup D_{\alpha}^{\prime}$ we obtain 3manifolds $M_{1}, M_{2}$ each with one boundary component homeomorphic to $\mathbb{S}^{2}$. By gluing 
a 3-ball along the boundary component of each, we obtain that $M$ is homeomorphic to $M_{1} \# M_{2}$ with $M_{1}, M_{2}$ having Heegaard genus $\leq g-1$.

\section{REFERENCES}

1. E. Akbas, A presentation for the automorphisms of the 3-sphere that preserve a genus two Heegaard splitting, Pacific J. Math. 236(2) (2008), 201-222.

2. Ch. Charitos, I. Papadoperakis and G. Tsapogas, A complex of incompressible surfaces and the mapping class group, Monatshefte für Mathematic, 167(3-4) (2012), 405-415. doi:10.1007/s00605-012-0379-8.

3. S. Cho, Homeomorphisms of the 3-sphere that preserve a Heegaard splitting of genus two, Proc. Amer. Math. Soc. 136(3) (2008), 1113-1123.

4. A. T. Fomenko and S. V. Matveev, Algorithmic and computer methods in 3-manifolds (Kluwer, Amsterdam, Netherlands, 1997).

5. L. Goeritz, Die Abbildungen der Brezelfläche und der Volbrezel vom Gesschlect 2, Abh. Math. Sem. Univ. Hamburg 9 (1933), 244-259.

6. W. Harvey, Boundary structure of the modular group, Riemann surfaces and related topics, in Proceedings of the 1978 Stony Brook conference, State University New York, Stony Brook, NY (Princeton University Press, Princeton, NJ, 1978) (Ann. Math. Stud. 97 (1981), 245-251).

7. A. Hatcher, P. Lochak and L. Schneps, On the Teichmüller tower of mapping class groups, J. Reine Angew. Math. 521 (2000), 1-24.

8. A. Hatcher and W. Thurston, A presentation for the mapping class group of a closed orientable surface, Topology 19(3) (1980), 221-237. 479.

9. D. Margalit, Automorphisms of the pants complex, Duke Math. J. 121(3) (2004), 457-

10. H. Masur and Y. N. Minsky, Quasiconvexity in the curve complex, in The tradition of Ahlfors and Bers, III, Contemporaty Mathematics, vol. 355 (American Mathematical Society, Providence, RI, 2004), 309-320.

11. D. McCullough, Virtually geometrically finite mapping class groups of 3-manifolds, $J$. Differ. Geom. 33(1) (1991), 1-65.

12. M. Scharlemann, Automorphisms of the 3 -sphere that preserve a genus two Heegaard splitting, Bol. Soc. Mat. Mexicana 10(3) (2004), 503-514 (special issue). 